\title{
A Journey from Abstract Film to Concrete Interaction
}

\author{
Ernest Edmonds
}

IOCT, Leicester Media School, De Montfort University, Leicester, UK

ernest@ernestedmonds.com

\begin{abstract}
The paper reviews abstract films and the notions of time that occur in them, contrasting them with the use of time in digital art. Developments by the author in making various generative digital abstract, or concrete, works are described and compared to film. The generation of the time element of the works described is integral with the generation of images. It is shown how different approaches to dealing with time in the digital context have emerged. In particular, an integrated constructivist approach has built from concepts in abstract film to go beyond cinema in a way that makes significant use of digital media. It has been possible to develop these works into interactive pieces by using artificial intelligent methods in various ways. True to the spirit of the early work described, these interactive works are also concrete rather than virtual. The art uses artificial intelligence to make real realities.
\end{abstract}

Keywords: abstract art; artificial intelligence; film; generative art.

\section{Introduction}

Today, film is mostly a digital, rather than a celluloid, medium. In parallel, digital art is increasingly time-based. The underlying paradigms, and the way that time is dealt with, are however quite different. From a technical perspective, Artificial Intelligence (AI) is becoming all pervasive, following computing itself. This is as true in the visual arts as anywhere else. In the popular press AI is often understood to simply be that branch of the subject that employs connectionist methods for machine learning and that has generated so many successful applications. This paper looks at a particular aspect of both of these developments, including a more general view of AI, through the lens of the author's own art. In contrast with much that we see, this work does not use artificial 
intelligence to construct, modify or display artificial worlds or situations but is firmly grounded in the idea of constructing concrete realities, in this case realities comprising interactive experiences of one kind or another. To explain this clearly, it is put into an historical context. This paper begins with the links between abstract digital art and abstract film.

The use of the word 'abstract' in art is a little odd. In ordinary language the noun 'abstract' refers to a reduced representation of something. An 'abstraction' would similarly normally refer to a form of representation. The art use of the term is in contrast with representational figurative paintings, which might be thought of as abstract representations of landscapes or people. For some reason the term was applied to art that, in fact, is an abstract of nothing at all. The movement towards abstract art, from Cézanne, through Cubism and to Kandinsky, Malevich and Mondrian, for example, was in a direction of increasingly sparse or distant abstraction from the subjects of figurative painting. Once the work ceased to reference external reality at all, and hence ceased to be an abstraction, the label 'abstract', inappropriately, stuck.

Lawrence Alloway defined useful ways of handling the problem: "I propose to use abstract meaning to draw out of or draw away from. Figurative paintings and landscapes will be said to have been abstracted from figures and landscapes. The word concrete will be used for works of art in which a process of abstraction is not perceptible.... I am aware of the logical objections to the term non-figurative but ... It is used here as an exclusive term for the whole field." (Alloway 1954).

Conny Dietzschold elaborates on concrete art, "this was the area of modern art which began in Europe around 1910, to detach itself entirely from the reproduction of the object ... the term 'concrete' is to be distinguished from 'abstract' - the latter denoting art which does not relinquish its relationship to reality. ... by definition it is the 
autonomy of pictorial means which constitutes Concrete Art, their insistence that the pictorial world is a world in and of itself, founded on surface, space, line, colour, light, light/dark contrasts and movement." (Dietzschold 2005).

Concrete art is concerned very directly with reality. It is concerned with the reality of the art object and our perception of it. Through challenging perception and making concrete art that addresses the very substance of surface, colour, movement and so on, the artist is extending our language and hence constructing new realities. If a work changes in time, as a film does, for example, then the properties of the transitions in time are part of the concrete reality of that work.

A significant proportion of digital art is time-based. It may interact with the environment and/or the audience or it may simply change over time in the manner of film. In any case, time is often an important factor in digital works. Strangely, perhaps, in discussing digital art we seem to put more emphasis on, for example, the graphical displays, the sounds and the modes of interaction than time itself. Music is, of-course, a classic time-based medium and film also has a serious history. In both cases, time receives significant attention.

Having discussed the concrete/abstract issue we can continue to use the term 'abstract' in what follows in its normal art meaning but being clear that the material reality of the art object rather than some abstracted representation of the world is what the paper is concerned with.

\footnotetext{
Abstract Film

Various time-based media have been important in leading to current developments in digital art. A concern for the media themselves and their formal concrete properties has a significant history that is relevant to current developments. Looking back over his work in film, for example, Hans Richter characterized his practice in terms of the
} 
organization of abstract forms and said this was about "a universal language, which is what abstract art should be about" (Foster 1998). This was not a new position to take. Indeed, Cézanne was reported to have said, "The technique of any art consists of a language and a logic" (Larguier 2001: 17). This concern for the concrete in art was not confined to painting and Richter was not the only interested filmmaker. For example, in discussing Vertov's movies, Malevich said, "Here, ... the elements are not shackled together in a whole in order to convey the gossip of existence ... He shows the movement itself, the dynamics whose force has been previously overshadowed by the cigarette-holder..." (Malevich 2002). In fact, Vertov "wanted not only to demonstrate the material and constructed nature of film, but also the reality constructed with it." (Weibel 1979).

Malcolm Le Grice published his book “Abstract Film and Beyond” in 1977 (le Grice 1977). This was an important and thorough review of developments up to that time of film as a concrete medium. The concerns covered in this book were not those of film as a vehicle for story telling but with the film medium itself. Abstract film began with a non-representational position, which was drawn largely from painting, rather than theatre. This distinction was discussed in the early days, for example, in the writings of Malevich (Bulgakowa 2002). As one might expect from his painting, he strongly supported the idea of abstract film (without calling it that).

The first abstract films date from 1910 to 1912. They were made by Bruno Corra and Arnaldo Ginna. Cora described their exploration as a series of artistic experiments that had quite varied levels of success: “...after the entire theory had been minutely established, we decided to make a serious attempt to create a music of colours. We immediately began to think of the instruments, which perhaps did not exist, .... We turned our thoughts to cinematography, and it seemed to us that this medium, slightly 
modified, would give excellent results, since its light potency was the strongest one could desire. The other problem concerning the need to have hundreds of colours at our disposition was also resolved, since, by exploiting the phenomenon of the persistence of an image on the retina, we would indeed have been able to make many colours merge, in our eye, into a single hue. To achieve this it was sufficient to pass all the component colours in front of the lens in less than a tenth of a second. In this way with a simple cinematographic instrument, with a machine of small dimensions, we would have obtained the innumerable and extremely powerful effects of large musical orchestras, the true chromatic symphony..." (Corra 1912).

Peter Kubelka’s “Arnulf Rainer” from 1960 demonstrated abstract film in its most pure concrete form (Kubelka 1960). It contains only pure white and pure black frames. It is “... a constructivist work, a visual music that develops a pattern of relationships between the periods of black screen and those of white..." Kubelka treated time in the manner of music and related it to constructivist art.

The relationship of abstract film to music seems obvious and it is not surprising to read Cora's and Kubelka's descriptions using musical terms. However, the concern moved on, as Le Grice described it, to explore the concrete medium itself in its own terms. Thus film has very particular properties. For example, it employs a fixed number of frames that are shown in any one second. Altogether, a significant issue in abstract film is the manipulation of time, including the relationship between time in the recording (or filming) process to time in projection. These can be quite different and changes between one and the other are often used as a significant feature of a film's properties.

\section{Film and Technological Innovations}

Le Grice mentions computer generated abstract films that had by then been produced, 
such as "Random" by Mark Adrian. These were made frame-by-frame, of-course. One might say that the computer acted as a very fast animator, generating frames at whatever pace was convenient for recording, with the actual pace of the film realized at the time of projection.

As Le Grice published his book technological change was rife. From the introduction of Sony's Portapak in 1965 (Boyle 1992), the new medium of video recording emerged. Whist similar in many ways to film, its differences were such that the genre of "video art" developed in a direction distinct from film. This genre has remained to this day, notwithstanding the extensive incorporation of video recording technologies into film making. At the same time, it is hard to see a feature film today that has not also used computer generated elements. Today, branching structures are often implemented in computer controlled films to make them interactive. This approach has been investigated by experimental film makers, such as Mike Leggett, introducing novel interaction devices to extend the medium of film, bringing it closer to interactive art (Leggett 2011). The technologies have merged but, in terms of the concrete time-based arts, the distinctions still matter as are explored below.

Incorporating computers into artworks has extended the possibilities still further with the option, for example to make works that change for ever and that do not have to exist within a fixed time period.

Computer-based generative art was first simply seen as algorithmic, i.e. art produced with the aid of a computer by programming it to follow some procedure that generated the art object. Today, such processes are most often associated with timebased art in which the generation of images is seen as a projection over time by the audience or viewer. For a full discussion of generative art see the author's co-written book (Boden and Edmonds 2019). Often, as in much of the author's work, the 
algorithmic element is dealt with by using declarative, rather than procedural, programming. In such cases the declarative description states what is required in terms of rules and it is left to the computer system to work out the order in which things should be done

\section{Generative Video Art}

The paper is concerned with the considerations of time in concrete, abstract, film making and with computer generated images and video recording from the early $1980 \mathrm{~s}$ and with the implications that remain today. In particular with the implications for art that uses artificial intelligence.

Between 1980 and 1985 the author developed a system for making time based abstract artworks that were generated by computer in real time (i.e. in the time employed for projection) and recorded directly onto videotape. The first completed piece was "Fragments" which lasted just over one hour and was shown, looping, as part of an exhibition at Exhibiting Space, London, in 1985. See figures 1,2,3,4. The images consisted of various arrangements of black and white squares as well as pure black and pure white frames. The key point about this work was the full incorporation of the time element into the generative process implemented within the computer. Time was a concrete part of the constructed work.

In animation, one can see the film as an ordered set of images, that may or may not be computer generated. Time is then imposed on the order by allocating a period for each images to be displayed and hence determine how many identical frames should be generated. In this sense, time is an independent aspect of the film. The timeline may be composed separately from the image design. In addition, the time in recording or filming need have no direct relationship with projection time. 
In the approach used for "Fragments", however, the generation of images and the timings are integrated. A generative computer program was used to define the work and produce it as computer output in real time. Rather than recording frames on film, the output from the computer was fed directly into a Umatic video recorder. In the computer program, a set of generative rules were defined that specified a search through a space of image possibilities. The space was a simple grid in which squares could either be black or white. The searching method is based on backtracking in logic programming (Edmonds 1988). The time structures of the work are an integral part of the searching process.

In "Fragments", and other work done at that time, both the images and the timing are determined by the generative rules as the computer system works through them. The totality of the work, therefore is completely implicit in the defining rules.

It was noted that Kulbelka said of his work "Arnulf Rainer" that it was "...a constructivist work, a visual music that develops a pattern of relationships ...". "Fragments" did exactly that, but because it was a computer generated work, as described above, the pattern of relationships over time were literally constructed in real time as the piece evolves. Another factor in this piece was that as there was no need to build it frame by frame it could be much longer than abstract films have typically been. It was shown in a gallery, rather than a cinema and people could come and go, watching just a part of it. It was interesting, however, that several interested artists were seen to watch the full two hours.

The images used in "Fragments" were deliberately simple because the complexity of change in time was felt to be sufficient. Additionally, the technology of colour screens was not reliable, and therefore no colour was used. Soon afterwards technology improved in that respect, with colour calibration becoming more available. 
A work that used a limited colour range was made and shown in Rotterdam in 1989. That work was called "Sydney”. See figures 5,6,7. It lasted just 15 minutes. It was produced in exactly the same way as "Fragments" but with a more populated grid and, of-course, generative rules for determining colour choices as well as the other aspects of the work. Thus "Sydney" was also a constructivist work with time, shape and colour relationships developed from a single set of generative rules. Today, of-course, the technology allows for considerable more sophistication, but the same key concerns remain. Casey Rees, for example, uses machine learning software to make generative videos, such as the Early Delights series (Rees 2019), that also both generates the images in real time and is not constrained by a pre-determined duration.

\section{Coding Interaction}

More recent work has added input from image analysis systems and removed the notion of a fixed duration altogether, for example in my 2001 work "Kyoto" (Edmonds and Dixon 2001). Certain artificial intelligence methods are used to build the core of the resulting interactive art works. This is something that the author has been concerned with for a very long time (Cornock and Edmonds 1973). AI has employed some very different approaches that are broadly divided into first and second wave periods. 1973 was within the first wave and in 2001, after a low time for AI, the second wave was just beginning to grow towards its very prominent position today, when $\mathrm{AI}$ is often seen as simply machine learning. For a full discussion, see, for example, Brian Cantwell Smith (Smith 2019). In the work covered here methods from both waves are relevant. Ofcourse, the incorporation of the analysis of motion rules in an artwork required a computer to control it and so ruled out the use of videotape. However, by showing the works on high quality displays, suitably mounted, a further step was taken in relation to time. The generative process may continue effectively for ever. As the rules are 
constantly being revised we have ever changing works that can exist over long time periods and, in that sense go beyond time, as it is used in film. They also go beyond the film paradigm in that they are "living" works. As they interact with participants and with the world around them they respond, change and develop.

AI is used to drive these processes in two main respects. First, by taking data (such as images) from the world, the art work's software looks for patterns and makes some kind of deduction about what it "perceives". Second, the collection over time of "perceived" entities is used to influence both the behaviour and the development of the work.

The coding of these different elements of such works can use different approaches. In input data analysis it is possible to use traditional algorithmic pattern analysis, such as applying the BitBLT Boolean function, for general properties. However, connectionist, second wave, AI methods provide significantly stronger recognition capability. But, these connectionist techniques are less helpful for the core code used to influence the artwork's behaviour. The reason is that, in developing the interactive artwork, it is important to explore, prototype and interrogate the artificial intelligence code as it evolves. Coding the way in which "perception" influences the direct behaviour and the behaviour rules of the work is at the core of making such an artwork. The artist needs to consider in detail exactly how, why and when changes are made, Hence, the difficulty of interrogating a connectionist system makes it hard to use. Symbolic, first wave, AI methods are much more congenial here. We can see that the AI coding used in this context is quite likely to be hybrid, employing both symbolic and connectionist methods (Edmonds 2018).

\section{Constructive Interaction}

In a series of such works known as "Shaping Form" a camera is placed near the monitor 
and the computer constantly analyses the movements of people in front of the work (see figure 8). Rather than leading to direct interaction, the computer system builds up a history of the movements and slowly modifies the generating rules being applied. In this way, the "Shaping Form" works are always changing, using new sets of colours and modifying the pace of change (Edmonds 2007a). The "Shaping Form" series consists of unique abstract interactive artwork that are each generating colours and forms in time from a set of unique rules: rules that are rather like their DNA. But they also take data from a camera and continuously calculate the amount of activity seen in front of the work. The computer software then steadily modifies the rules according to an artificial intelligence program that is at the heart of the artwork. The development over time is, then, influenced by the people who look at it.

"Shaping Form" is a representation of computed life, moving and changing of its own accord but maturing and developing as a result of the movement of audiences. The monitor shows a living matrix of colours that sometimes change very slowly and at other times burst into life, depending on the movement of people in the space. The colours used are from a small, but changing, pallet of hues at any one time but the saturation and brightness levels can vary considerable. Images are generated using rules that determine the colours, the patterns and the timing. People can readily detect the immediate responses of the work to movement but the changes over time are only apparent when there is more prolonged, although not necessarily continuous, contact with it. A first viewing followed by one several days, or even months, later will reveal noticeable developments in the colours and patterns.

Digital artworks like "Shaping Form" are designed to interact with the environment in which they are found. The works is made to learn from external movement such as a hand waving or a person walking by. The way it 'learns' 
determines the choice of colour and pattern in the images displayed as well as the timing of changes. The behaviour of the work is not intended to always be obvious, so that if you continuously try to force a response by waving or shouting, that might result in a period of stillness.

The audience help to shape the work. "Shaping Form" is a representation of computed life, moving and changing of its own accord but maturing and developing as a result of the movement of audiences. The shaping of the form is a never-ending process of computed development. Each unique work is identified by the date included in its title. That date is the day that it began its operation, when it came alive.

As has been discussed elsewhere (Edmonds 2007b), the processes of interacting with the world being used in artworks such as "Shaping Form" do not fall into the more common category of game-like interactive systems. The artwork changes its nature over long periods of time in response to events. It is influenced over time, one might say, rather than acting in a direct interaction mode. Equally, the interaction is not with a virtual world, or a representation of anything but, as with the abstract films discussed above, with the concrete reality of a monitor and the colours that it displays. The artificial intelligence is not driving an artificial reality seen through some device but a concrete reality experienced directly. The nature of this experience has been investigated in relation to an installation, "Shaping Space", that extends the concepts in the "Shaping Forms" (Ximena et al 2014).

An extensive review of the authors work is provided in the book by Francesca Franco (Franco 2017).

\section{Conclusion}

The paper reviewed aspects of abstract films and the notions of time that occur in them. The interest in the concrete reality of the film, rather than its narrative, is the abstract 
aspect that is carried over into the digital works discussed. The specific role of time is then emphasized. A series of developments by the author in making various generative digital abstract works have been described and compared to film. The generation of the time element of the works described is integrated with the generation of images. It has been shown how different approaches to dealing with time in the digital context have emerged. Most significantly, it has been possible to develop these works into interactive pieces by using artificial intelligent software in various ways. True to the spirit of the early work described, these interactive works are also concrete rather than virtual. The art uses AI to make real realities.

\section{Acknowledgements}

Parts of this paper are drawn from an earlier conference presentation by the author (Edmonds 2010). The author is grateful to the anonymous reviewers of the first draft, who made a number of valuable suggestions.

\section{References}

Alloway, L. 1954. Nine Abstract Artists. Tiranti, London, Boyle, D 1992. "From Portapack to Camcorder: a Brief History of Guerrilla Television". Journal of Film and Video, 44, 1/2. pp 67-79.

Bulgakowa, O (ed) 2002. Kazimir Malevich: The White Rectangle, Writings on Film. Potemkin Press, San Francisco, CA.

Cornock, S. and Edmonds, E. A. 1973. "The creative process where the artist is amplified or superseded by the computer". Leonardo, 16, pp 11-16.

Corra, B. 1912. "Il pastore, il gregge e la zampogna". English translation, "Abstract Cinema-Chromatic Music", in Apollini, U. (editor) (2009) Futurist Manifestos. Tate Publishing, London. Also available at http://www.unknown.nu/futurism/abstract.html.

Dietzschold, C. 2005. “Opening Speech” Minimal Approach...Concrete Tendencies. Tin Sheds Gallery, University of Sydney, April 2005. 
Edmonds, E. A. 1988. "Logic and time-based art practice." Leonardo, Electronic Art Supplemental issue, pp 19-20.

Edmonds, E. A. 2007a. "Shaping Form Series”. In Jennings, O. (editor) Speculative Data and the Creative Imaginary. National Academy of Sciences, Washington DC. pp 18-9.

Edmonds, E. A. 2007b. "Reflections on the Nature of Interaction". CoDesign. 3, 3. pp 139-143.

Edmonds, E. A. 2010. "Beyond abstract film: constructivist digital time" Proceedings Electronic Visualisation and the Arts, BCS, London.

Edmonds, E. A. 2018. "Algorithmic Art Machines." Arts 7, no. 1: 3. http://www.mdpi.com/2076-0752/7/1/3

Edmonds, E. A. and Dixon, J. 2001. "Constructing Inter-relationships: Computation for Interaction in Art". Gero, J. S. and Maher, M. L. (eds) Computational and Cognitive Models of Creative Design V. Key Centre of Design Computing and Cognition, University of Sydney. pp 173-156.

Foster, S. C. (editor) 1998. Hans Richter: Activism, Modernism and the Avant-Garde, MIT Press, Cambridge, MA.

Franco, F. 2017. Generative Systems Art: The Work of Ernest Edmonds. Routledge, London.

Kubelka, P. 1960. Arnulf Rainer. YouTube version at http://www.youtube.com/watch?v=iw1DVtFAz64

Larguier, L. 2001. Conversations with Cézanne, edited by Michael Doran, University of California Press.

Leggett, M. 2011. "Memory, Schema and Interactive Video". In Candy, C. and Edmonds, E. (eds) 2011. Interacting: Art, Research and the Creative Practitioner. Libri Publishing. Faringdon, Oxfordshire. pp 282-294.

Le Grice, M. 1977. Abstract Film and Beyond. Studio Vista, London.

Malevich, M. 2002. "Pictorial Laws in Cinematic Problems". In Bulgakowa, 2002. pp 77-84.

Rees, C. 2019. Earthly Delights. http://www.reas.com/earthly_2/.

Smith, B. C. 2019. The Promise of Artificial Intelligence: Reckoning and Judgement. MIT Press, Cambridge, MA.

Weibel, P. 1979. “Eisenstein, Vertov and the Formal Film”. In Film as Film, Hayward Gallery, London, pp 47- 51. 
Ximena, A., Askaroff, K., Candy, L., Edmonds, E. A., Faram, J. and Hobson, G. 2014. "Evaluation in Public Art: The Light Logic Exhibition". In Candy, L. and Ferguson, A. (editors) Interactive Experience in the Digital Age: evaluating new art practice. Springer, London. pp 187-208. 